\title{
Next-generation COVID-19 vaccines: Opportunities for vaccine development and challenges in tackling COVID-19
}

\author{
Qian $\mathrm{Li}^{1, \S}$, Jun Wang ${ }^{2, \S}$, Yang Tang ${ }^{1, \S}$, Hongzhou $\mathrm{Lu}^{1, *}$ \\ ${ }^{1}$ Department of Infectious Diseases, Shanghai Public Health Clinical Center, Shanghai, China; \\ ${ }^{2}$ Center of Clinical Laboratory, The Fifth People's Hospital of Wuxi, Jiangnan University, Wuxi, China.
}

SUMMARY The ongoing COVID-19 pandemic, caused by severe acute respiratory syndrome coronavirus 2 (SARS-CoV-2), has become a global threat. Although non-pharmaceutical interventions have been rigorously and widely implemented, living conditions caused by the pandemic will last until highly effective vaccines are successfully improved and globally administered. Several first-generation COVID-19 vaccines were approved at the end of 2020. However, the COVID-19 pandemic is persisting worldwide. To be clear, the efficiency and the coverage of current vaccines are insufficient, but newly emerging and rapidly spreading variants are the most pressing concern. A second-generation COVID-19 vaccine worth mentioning, NVX-CoV2373, has demonstrated 90\% overall efficacy as well as a high level of efficacy against circulating variants in Phase 3 clinical trials. Currently, NVXCoV2373 is the only vaccine that has proven successful against variants during Phase 3/4 trials. Therefore, developing the next generation of vaccines is a promising strategy to ultimately prevail against SARS-CoV-2. This review provides up-to-date information on COVID-19 vaccines in terms of their efficacy and new platforms and the progression of COVID-19 vaccination. Moreover, this review also summarizes the efficacy of approved COVID-19 vaccines against variants. Lastly, this review highlights the global challenges for COVID-19 vaccines in development and vaccination, and it discusses opportunities for development of future COVID-19 vaccines and vaccination coverage.

Keywords COVID-19, SARS-CoV-2, vaccines, NVX-CoV2373, vaccination, distribution

\section{Introduction}

As of June 25, 2021, more than 17 million confirmed cases of COVID-19 and 3,840,223 deaths have been reported by the WHO (1). The COVID-19 pandemic has posed a serious crisis to both health care systems and economies worldwide. Since there are no effective treatments for COVID-19, the chances of controlling the COVID-19 pandemic depend mainly on two main factors: $i$ ) public health interventions and ii) development and administration of safer and more effective vaccines (2).

Public health interventions such as nonpharmaceutical measures were obviously effective in reducing the spread of COVID-19 (3). Governmental measures including travel restrictions, border restrictions, quarantine of travelers, confirmed cases, and contacts, orders to avoid confined spaces and large gatherings, social distancing, compulsory mask wear, school closures, and establishment of designated hospitals were useful at preventing the spread of COVID-19. Individual interventions, such as use of protective equipment by healthcare workers and attention to personal hygiene, were also effective in tackling COVID-19. Nevertheless, sporadic cases of COVID-19 continue to emerge even in countries with strict controls compared to countries with less stringent interventions $(4,5)$.

More importantly, a number of newly emerged mutations have accelerated the rapid spread of SARSCoV-2. There are 4 known major variants: the B.1.1.7 lineage (called the Alpha variant) that was first identified in the United Kingdom, the B.1.351 lineage (called the Beta variant) that was identified in South Africa, the P.1 lineage that was identified in Brazil (called the Gamma variant), and the B.1.617 lineage (called the Delta variant) that was verified in India. These variants have been labeled variants of concern (VOC) by the WHO (6). At the current point in time, the B.1.617 variant has been blamed for the current surge of COVID-19 in India (7). The variants have increased transmissibility or increased virulence compared to the original virus. Changes in those variants cause worse disease presentation and negatively impact COVID-19 epidemiology and public health measures. Therefore, strict control measures 
alone are not effective enough to stop the COVID-19 pandemic, more efficacious vaccines need to be quickly developed to prevent the COVID-19 pandemic.

\section{Strategies for the development of COVID-19 vaccines}

As of June 25, 2021, a total of 574 vaccines have been developed, including 103 vaccines in clinical trials, and 184 vaccines in pre-clinical studies. Of the 103 vaccines in clinical trial, 23 are in Phase 3 or 4. Multiple platforms have been used to develop the 103 vaccines. Eighteen are inactivated and live attenuated vaccines, including BBIBP-CorV (COVILO) from Sinopharm (Beijing and Wuhan) and CoronaVac from Sinovac (8). Thirty-three are recombinant protein vaccines, or recombinant subunit vaccines, such as NVX-COV2373 from Novavax and ZF2001 from Zhifei Biology. Twenty-six are nucleoid vaccines, such as BNT-162b2 from BioNTech and mRNA-1273 from Moderna. Twenty-one are viral vector vaccines, such as ChAdOx1-nCoV-19 from AstraZeneca, Ad5-nCoV from CanSino, Ad26.COV2.S from Janssen Pharmaceuticals, and Sputnik V from Gamaleya (9).

Vaccines developed using different platforms have pros and cons because of the different techniques used $(10,11) . i)$ Inactivated vaccines and live attenuated vaccines are quick to prepare and easy to produce but do not readily induce T-cell immunity and usually need to be administered 2-3 times to enhance immunity. In addition, the long-lasting immune response to inactivated vaccines relies more on adjuvants than other vaccines, and live attenuated vaccines against complex pathogens are challenging (12-13). ii) Nucleoid vaccines are easy to rapidly mass produce and confer long-term host immunogenicity but have potential genetic risks since mRNA is unstable and they remain ineffective when administered to humans with compromised immunity (14-15). iii) Viral vector vaccines can induce strong humoral and cellular immunity but are not able to induce immunogenicity in the face of preexisting immunity (1618). iv) Recombinant vaccines such as NVX-COV2373 from Novavax and ZF2001 from Zhifei Biology have very clear advantages such as a distinct composition and a high level of safety and stability but also have weak immunogenicity and require adjuvants (19). (Table 1)

\section{Progress of COVID-19 vaccination}

Widespread vaccination is also required to end the COVID-19 pandemic. In mid-December 2020, the first reports of a COVID-19 vaccine outside of clinical trials were published in the UK, thus sparking a race for vaccine development. Waves of vaccination subsequently occurred daily around the world. With the successful development of the first-generation vaccine, the COVID-19 vaccination rate increased in a wide range of countries from January to May 2021 (20).
Thanks to the tremendous breakthrough in COVID-19 vaccine development, the WHO is working tirelessly with partners to manufacture vaccines and to promote their safety and effectiveness. As of June 25,2021 , a total of $471,786,361$ persons were fully vaccinated and 1,031,602,050 persons were vaccinated with at least one dose, accounting for $22.4 \%$ of the world's population $(1,21)$. Two-point-eight billion doses have been administered, and 40.8 million doses are administered each day (21). However, the vaccination rate $(>$ and $=2$ nd dose) among people in low-income countries was less than $1 \%$, which implies that the ongoing COVID-19 vaccination faces great challenges.

\section{Challenges with COVID-19 vaccines in development and vaccination}

\subsection{Issues with the efficacy of COVID-19 vaccines against variants}

SARS-CoV-2 is an enveloped virus with a positivepolarity single-stranded RNA genome that contains four major structural proteins: the spike (S), membrane (M), envelope $(\mathrm{E})$, and nucleocapsid $(\mathrm{N})$ proteins. The trimeric $\mathrm{S}$ protein consists of a receptor-binding subunit (S1) and a membrane-fusion subunit (S2). The $\mathrm{S} 1$ subunit consists of the N-terminal domain (NTD), the receptor-binding domain (RBD), and two small subdomains. The role of the $\mathrm{S}$ protein is to mediate SARS-CoV-2's entry into host cells via surface receptor angiotensin-converting enzyme 2 (ACE2). The S1 subunit is involved in cell entry, and the RBD domain is responsible for direct binding. Thus, the $\mathrm{S}, \mathrm{S} 1$, and RBD proteins are 3 major targets for vaccine development (22-24).

However, mutations in those target proteins represent a great challenge to the efficacy of COVID-19 vaccines (25). As reported, variants of concern (VOC) are associated with increased transmissibility and virulence due to notable mutations in key proteins. For example, mutations in the B.1.1.7 lineage involve multiple sites, such as a N501Y substitution in the RBD region, H69/V70 deletion in the N-terminal region, and $\mathrm{P} 681 \mathrm{H}$ mutation adjacent to the furin cleavage site in the $\mathrm{S}$ protein. Mutations in the B.1.351 variant (20H/501Y.V2) include K417N, E484K, and N501Y. In the P.1 variant (B.1.1.28.1), mutations involve K417T, E484K, and N501Y substitutions in the RBD domain. B.1.617.2 is defined by more mutations in the $\mathrm{S}$ protein, including T19R, DEL157/158, T478K, and D950N. In addition, these variants share the D614G mutation, which has been found to increase the rapid spread of the virus. Therefore, inadequate public healthcare measures and vaccination coverage have accelerated the emergence of variants. Moreover, the rapid spread of variants has increased the global demand for more effective vaccines. Whether the 6 prototype vaccines in Phase III/IV trials will remain effective against variants 


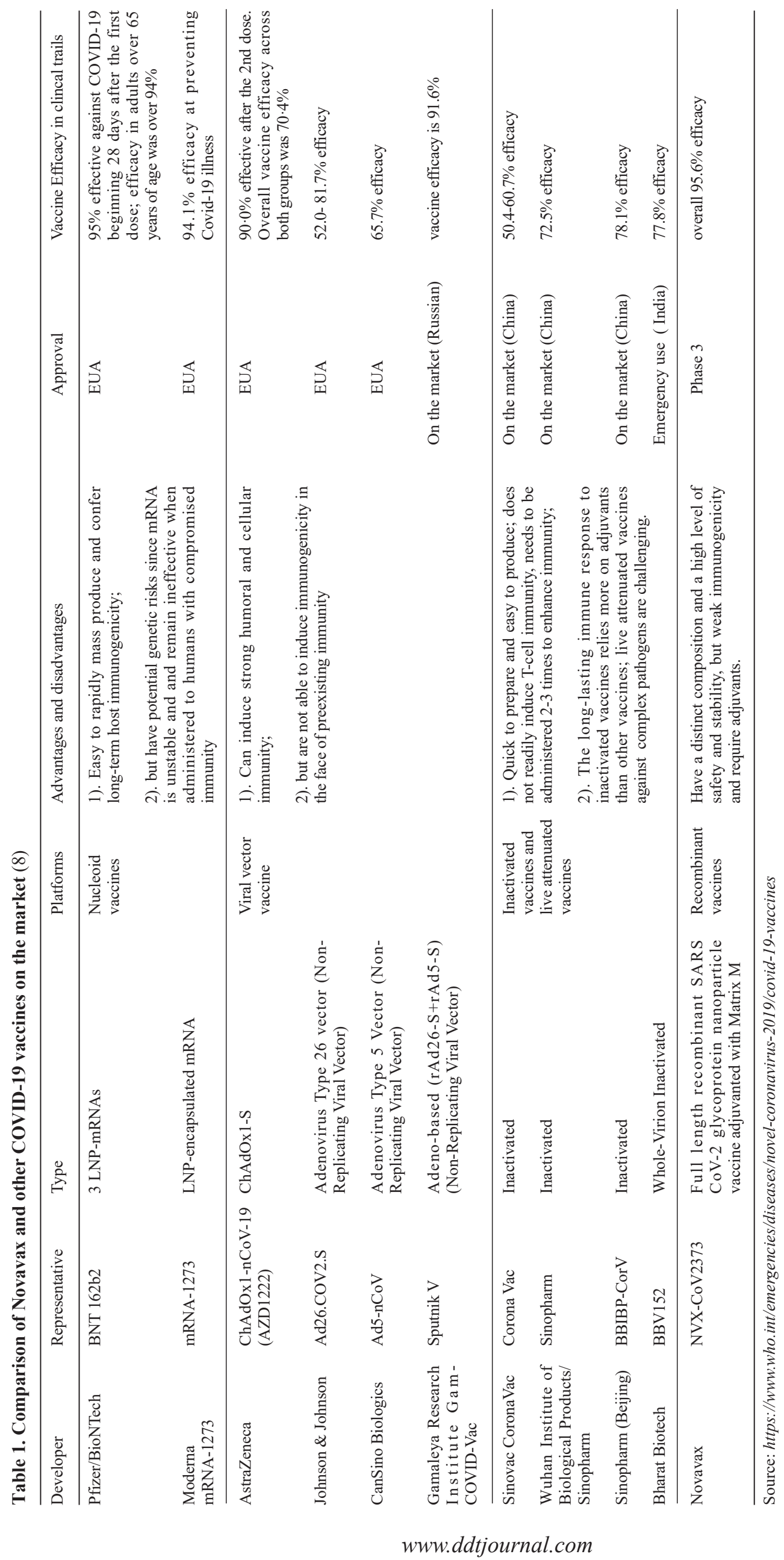


Table 2. Vaccine efficacy against variants of concern (5)

\begin{tabular}{|c|c|c|c|c|c|}
\hline WHO label & Lineage & $\begin{array}{l}\text { First discovery } \\
\text { place }\end{array}$ & $\begin{array}{c}\text { Date of } \\
\text { confirmation }\end{array}$ & Mutations & $\begin{array}{l}\text { Vaccine Efficacy } \\
\text { aginast variants }\end{array}$ \\
\hline Alpha & B.1.1.7 & United Kingdom & $18-12-2020$ & $\begin{array}{l}\text { N501Y substitution in the RBD region, H69/ } \\
\text { V70 deletion in the N-terminal region, and } \\
\text { P681H mutation adjacent to the furin cleavage } \\
\text { site in the S protein. D614G mutation }\end{array}$ & $\begin{array}{l}\text { NVX-CoV2373 } \\
(85.6 \%)\end{array}$ \\
\hline Beta & B.1.351 & South Africa & $18-12-2020$ & K417N, E484K, N501Y, and D614G mutation & $\begin{array}{l}\text { NVX-CoV2373 (49.4\%) } \\
\text { ChAdOx1 nCoV-19 (10\%) }\end{array}$ \\
\hline Gamma & P.1 & Brizal & 11-01-2021 & $\begin{array}{l}\text { K417T, E484K, and N501Y substitution in } \\
\text { the RBD domain, and D614G mutation }\end{array}$ & unknown \\
\hline Delta & B.1.617.2 & India & $\begin{array}{l}11-05-2021 \\
\text { labled as VOC }\end{array}$ & $\begin{array}{l}\text { more mutations in the spike protein, namely } \\
\text { T19R, DEL157/158, T478K and D950N, } \\
\text { D614G mutation }\end{array}$ & unknown \\
\hline
\end{tabular}

Source: https://www.ctvnews.ca/health/coronavirus/b-1-617-variant-first-identified-in-india-classified-as-variant-of-global-concern-1.5421363

is also a great concern (26). (Table 2)

\subsection{The challenges of globally promoting and} distributing vaccines

As of June $25,2021,60 \%$ of people are fully vaccinated against COVID-19 in Israel, and only $4.3 \%$ were partly vaccinated. A high level of vaccination coverage was noted in China ( $51 \%$ had received the $2^{\text {nd }}$ dose, $13 \%$ had received the $\left.1^{\text {st }}\right)$, the United Kingdom $(47 \%$ had received the $2^{\text {nd }}$ dose, $17 \%$ had received the $\left.1^{\text {st }}\right)$, and the United States ( $45 \%$ had received the $2^{\text {nd }}$ dose, $8.1 \%$ had received the $1^{\text {st }}$ ). The cumulative number of doses administered per 100 people was 123.44 in Israel. In some areas, however, such as South Africa countries, the total number of vaccinated per 100 people was less than $1(21)$. The large differences in the rate vaccination coverage between countries imply significant inequality in vaccine distribution, which may be caused by different national economics, difficulties with logistics and storage, lower rates of vaccination, the extent to which vaccination has been promoted by the government (27).

Moreover, different countries have taken markedly different approaches to vaccination. European Union countries like the United Kingdom have adopt a 'first dose first' approach. This vaccination strategy priority promotes wider coverage with the first dose while delaying administration of the second dose. Other countries, such as Israel, the United States, and China taken the approach of fully vaccinating smaller populations first. Different approaches also led to different rates of vaccination coverage among different populations worldwide $(20,28-31)$. The rapid development of COVID-19 vaccines within one year has been a great breakthrough in scientific and economic cooperation among countries. However, now that vaccines are being developed, the question is whether the global distribution of vaccines can match the speed of the COVID-19 pandemic, i.e. whether those vaccines can be administered quickly and widely distributed around the world (32).

\section{Strategies for development of future COVID-19 vaccines and vaccination coverage}

5.1 Optimizing the vaccine platform, learning from Novavax

Thus far, only a few clinical trials have investigated the effects of COVID-19 vaccines on variants. A phase 3 trial was conducted in South Africa to assess the efficacy of a single dose of the Ad26.COV2.S vaccine (Johnson \& Johnson/Janssen). Efficacy was reported to be $52 \%$ at 14 days and $64 \%$ at 28 days after the first dose of Ad26. COV2.S vaccine. The trial was contemporaneous with $95 \%$ of subjects being infected with the B.1.351 variant, but no vaccine is reported to be effective against the B.1.351 variant $(33,34)$. Another phase 2 trial was also conducted in South Africa to evaluate the efficacy of the ChAdOx1 nCoV-19 vaccine (AstraZeneca). However, overall efficacy was only $22 \%$ and efficacy against the B.1.351 variant was only $10 \%(35)$.

Most recently, the efficacy of the NVX-CoV2373 vaccine against variants was examined in a phase III clinical trial. Results indicated that the efficacy of the NVX-CoV2373 vaccine against SARS-CoV-2 was $95.6 \%$, that against the UK variant was $85.6 \%$, and that against the South African variant was $49.4 \%$. NVX-CoV2373 is the only vaccine effective against COVID-19 variants, but the next generation of vaccines will presumably be developed worldwide. A point worth noting is that the platform for NVX-CoV2373 used recombinant nanoparticle technology to generate an antigen from the spike (S) protein. The patented saponinbased Matrix-M $\mathrm{M}^{\mathrm{TM}}$ adjuvant in Novavax was designed to promote a humoral and cellular immune response (35). (Table 2)

The efficacy of NVX-CoV2373 in clinical trials implies that the next generation of vaccines, and especially protein vaccines, need to optimize their 
protein targets to enhance immunogenicity and to cover emerging and potential mutation sites. Moreover, appropriate adjuvants are useful at enhancing the immune response, and especially at inducing high levels of humoral immunity. As SARS-CoV-2 variants emerge, their surveillance, detection of neutralization antibodies, and the immune response to them in clinical trials are also crucial.

5.2. Optimizing vaccination strategies combined with non-pharmaceutical interventions

In rich countries such as Israel, promising vaccines are easier to develop and vaccination strategies are easier to optimize, and the country has done remarkably well with its vaccination efforts. In developing countries, however, vaccines are uncertain, so non-pharmaceutical interventions to combat COVID-19 was useful at limiting the spread of the virus and its variants. Under such conditions, the best strategies are social distancing, attention to personal hygiene, frequently handwashing, and mask wear while awaiting vaccines and affordable drugs (2).

Overall, vaccination strategies should be optimized in combination with non-pharmaceutical interventions to control the epidemic. Non-pharmaceutical interventions should be implemented as much as possible in developing or low-income countries. In the war against viruses, the faster the public health interventions and the more optimal the vaccine options, the more likely we are to win.

\section{Discussion}

Given problems with the efficacy of COVID-19 vaccines against variants, NVX-CoV2373 was a great scientific breakthrough, marking the first human victory in the battle against the virus and its variants. However, countries cannot rely on vaccines alone. Appropriate vaccination strategies and sufficient vaccination coverage, combined with non-pharmaceutical interventions, are key steps in controlling the global COVID-19 pandemic.

For a more complete and rapid future public health response to a disease like COVID-19, several aspects should be taken into account. First, the design of and clinical trials on next-generation vaccines have to take emerging variants into account. Second, countries where vaccines are lacking should to adopt a more flexible vaccination strategy since "administering a second dose of another vaccine is better than not administering one at all". As an example, further evidence is need to verify which types of vaccines can be mixed and which vaccines have sufficient safety and efficacy to cope with a temporary shortage of a given vaccine $(36,37)$. Third, special populations, including the elderly, infants, and immunocompromised patients such as those with AIDS or cancer, are not yet fully covered in clinical trials on
COVID-19 vaccines and should be considered in the future $(35,38)$.

Once COVID-19 vaccines are developed, widespread vaccine distribution and adequate vaccination coverage are also crucial steps. Thus, a plan for widespread vaccine distribution is needed based on different demographics, logistics, and acceptance and with the cooperation of various levels of government $(39,40)$. Lastly, non-pharmaceutical interventions should be implemented more rigorously, and especially in countries with insufficient COVID-19 vaccination coverage.

Funding: This work was supported by grants from the National Natural Science Foundation of China (grant no. 8207081611), the Shanghai Science and Technology Commission (KY-GW-2021-18/a hospital-level project by the Shanghai Public Health Clinical Center), and a grant from Top Talent Support Program for young and middle-aged people of Wuxi Health Committee.

Conflict of Interest: The authors have no conflicts of interest to disclose.

\section{References}

1. World Health Organization. WHO Coronavirus (COVID-19) Dashboard. https://covid19.who.int/ (accessed June 25, 2021).

2. Jun C, Hongzhou L. New challenges to fighting COVID-19: Virus variants, potential vaccines, and development of antivirals: The case of influenza viruses. Biosci Trends. 2021; 15:126-128.

3. Ayouni I, Maatoug J, Dhouib W, Zammit N, Fredj SB, Ghammam R, Ghannem H. Effective public health measures to mitigate the spread of COVID-19: A systematic review. BMC Public Health. 2021;21(1):1015.

4. World Health Organization. Tracking SARS-CoV-2 variants. https://www.who.int/en/activities/tracking-SARSCoV-2-variants/ (accessed June 25, 2021).

5. CTV News. B.1.617 variant first identified in India classified as variant of global concern. https://www. ctvnews.ca/health/coronavirus/b-1-617-variant-firstidentified-in-india-classified-as-variant-of-globalconcern-1.5421363 (accessed June 25, 2021).

6. World Health Organization. Overview of Public Health and Social Measures in the context of COVID-19. https:// www.who.int/publications/i/item/overview-of-publichealth-and-social-measures-in-the-context-of-covid-19 (accessed June 25, 2021).

7. Li Q, Wang L, Wang B, Lu H. The COVID-19designated hospitals in China: Preparing for public health emergencies. Emerg Microbes Infect. 2021; 10:998-1001.

8. World Health Organization. Coronavirus disease (COVID-19)/COVID-19 vaccines. https://www.who.int/ emergencies/diseases/novel-coronavirus-2019/covid-19vaccines (accessed June 25, 2021).

9. World Health Organization. COVID-19 vaccine tracker and landscape. https://www.who.int/publications/m/item/ draft-landscape-of-covid-19-candidate-vaccines (accessed June 25, 2021).

10. He Q, Mao Q, Zhang J, Bian L, Gao F, Wang J, Xu M, 
Liang Z. COVID-19 Vaccines: Current Understanding on Immunogenicity, Safety, and Further Considerations. Front Immunol. 2021; 12:669339.

11. Rawat K, Kumari P, Saha L. COVID-19 vaccine: A recent update in pipeline vaccines, their design and development strategies. Eur J Pharmacol. 2021; 892:173751.

12. Barría MI. Localized mucosal response to intranasal live attenuated influenza vaccine in adults. J Infect Dis. 2013; 207:115-124.

13. Kumar A, Meldgaard TS, Bertholet S. Novel platforms for the development of a universal influenza vaccine. Front Immunol. 2018; 9:1-14.

14. Kim JHJJ. DNA vaccines against influenza viruses. Vaccines Pandemic Influ. 2009; 333:197-210.

15. Rodríguez-Gascón A, del Pozo-Rodríguez A, Solinís MÁ. Development of nucleic acid vaccines: Use of selfamplifying RNA in lipid nanoparticles. Int J Nanomed. 2014; 9:1833-1843.

16. Geiben-Lynn R, Greenland JR, Frimpong-Boateng K, Letvin NL. Kinetics of recombinant adenovirus type 5, vaccinia virus, modified vaccinia ankara virus, and DNA antigen expression in vivo and the induction of memory T-lymphocyte responses. Clin Vaccine Immunol. 2008; 15:691-696.

17. Rollier CS, Reyes-Sandoval A, Cottingham MG, Ewer K, Hill AV. Viral vectors as vaccine platforms: deployment in sight. Curr Opin Immunol. 2011; 23:377-382.

18. Sekaly RP. The failed HIV Merck vaccine study: A step back or a launching point for future vaccine development? J Exp Med. 2008; 205:7-12.

19. Nascimento IP, Leite LCC. Recombinant vaccines and the development of new vaccine strategies. Braz J Med Biol Res. 2012; 45:1102-1111.

20. Mathieu E, Ritchie H, Ortiz-Ospina E, Roser M, Hasell J, Appel C, Giattino C, Rodés-Guirao L. A global database of COVID-19 vaccinations. Nat Hum Behav. 2021; doi: 10.1038/s41562-021-01122-8.

21. Our World in Data. Coronavirus (COVID-19) Vaccinations. https://ourworldindata.org/covidvaccinations (accessed June 25, 2021).

22. Wrapp D, Wang N, Corbett KS, Goldsmith JA, Hsieh CL, Abiona O, Graham BS, McLellan JS. Cryo-EM structure of the 2019-nCoV spike in the prefusion conformation. Science. 2020; 367:1260-1263.

23. Zeng $\mathrm{W}$, Liu G, Ma H, et al. Biochemical characterization of SARS-CoV-2 nucleocapsid protein. Biochem Biophys Res Commun. 2020; 527:618-623.

24. Huang Y, Yang C, Xu XF, Xu W, Liu SW. Structural and functional properties of SARS-CoV-2 spike protein: potential antivirus drug development for COVID-19. Acta Pharmacol Sin. 2020; 41:1141-1149.

25. World Health Organization. The effects of virus variants on COVID-19 vaccines. https://www.who.int/news-room/ feature-stories/detail/the-effects-of-virus-variants-oncovid-19-vaccines (accessed June 25, 2021).

26. Noh JY, Jeong HW, Shin EC. SARS-CoV-2 mutations, vaccines, and immunity: Implication of variants of concern. Sig Transduct Target Ther. 2021; 6:203.

27. Mills MC, Salisbury D. The challenges of distributing COVID-19 vaccinations. EClinicalMedicine. 2021; $31: 100674$.

28. Wouters OJ, Shadlen KC, Salcher-Konrad M, Pollard
AJ, Larson HJ, Teerawattananon Y, Jit M. Challenges in ensuring global access to COVID-19 vaccines: Production, affordability, allocation, and deployment. Lancet. 2021; 397,1023-1034.

29. Iacobucci G \& Mahase E. Covid-19 vaccination: What's the evidence for extending the dosing interval? BMJ. 2021; 372:n18.

30. Mahase E. Covid-19: Medical community split over vaccine interval policy as WHO recommends six weeks. BMJ. 2021; 372:n226.

31. Baraniuk C. Covid-19: How the UK vaccine rollout delivered success, so far. BMJ. 2021; 372:n421.

32. Li Q, Lu H. Latest updates on COVID-19 vaccines. Biosci Trends. 2021; 14:463-466.

33. Food and Drug Administration. Vaccines and Related Biological Products Advisory Committee, February 26, 2021, meeting announcement. https://www. fda.gov/advisory-committees/advisory-committeecalendar/vaccines-and-related-biological-productsadvisory-committee-february-26-2021-meetingannouncement\#event-materials (accessed June 25, 2021).

34. Johnson \& Johnson Announces Single-Shot Janssen COVID-19 Vaccine Candidate Met Primary Endpoints in Interim Analysis of its Phase 3 ENSEMBLE Trial. Press release, January 29, 2021.https://www.jnj.com/johnsonand-johnson-announces-single-shot-janssen-covid-19vaccine-candidate-met-primary-endpoints-in-interimanalysis-of-its-phase-3-ensemble-trial (accessed June 25, 2021).

35. Madhi SA, Baillie V, Cutland CL, et al. Efficacy of the ChAdOx1 nCoV-19 Covid-19 vaccine against the B.1.351 variant. N Engl J Med. 2021; 384:1885-1898.

36. Public Health England. Green Book. Chapter 14a: Covid-19-SARS-CoV-2. Dec 2020. https://assets. publishing.service.gov.uk/government/uploads/system/ uploads/attachment_data/file/948757/Greenbook_ chapter_14a_v4.pdf (accessed June 25, 2021).

37. Mahase E. Covid-19: Vaccine brands can be mixed in "extremely rare occasions," says Public Health England. BMJ. 2021;372:n12.

38. Saini KS, Martins-Branco D, Tagliamento M, Vidal L, Singh N, Punie K, Saini ML, Chico I, Curigliano G, de Azambuja E, Lambertini M. Emerging issues related to COVID-19 vaccination in patients with cancer. Oncol Ther. 2021; 16:1-11.

39. Wang W, Wu Q, Yang J, Dong K, Chen X, Bai X, Chen X, Chen Z, Viboud C, Ajelli M, Yu H. Global, regional, and national estimates of target population sizes for covid-19 vaccination: descriptive study. BMJ. 2020;371:m4704.

40. Wang J, Jing R, Lai X, Zhang H, Lyu Y, Knoll MD, Fang H. Acceptance of COVID-19 Vaccination during the COVID-19 Pandemic in China. Vaccines (Basel). 2020; $8: 482$.

Received June 16, 2021; Revised June 28, 2021; Accepted June 30, 2021.

${ }^{\S}$ These authors contributed equally to this work.

*Address correspondence to

Hongzhou Lu, Shanghai Public Health Clinical Center, Fudan University, 2901 Caolang Rd, Shanghai 201508, China.

E-mail: luhongzhou@fudan.edu.cn 